\title{
Estimating the impact of influenza on the epidemiological dynamics of SARS-CoV-2
}

\author{
Matthieu Domenech de Cellès ${ }^{\text {Corresp., } 1}$, Jean-Sébastien Casalegno ${ }^{2,3}$, Bruno Lina $^{2,3}$, Lulla Opatowski ${ }^{4,5}$ \\ 1 Infectious Disease Epidemiology Group, Max Planck Institute for Infection Biology, Berlin, Germany \\ 2 Laboratoire de Virologie des HCL, IAl, CNR des virus à transmission respiratoire (dont la grippe) Hôpital de la Croix-Rousse F-69317 Lyon cedex 04 , \\ France, Lyon, France \\ 3 Virpath, Centre International de Recherche en Infectiologie (CIRI), Université de Lyon Inserm U1111, CNRS UMR 5308, ENS de Lyon, UCBL F-69372, Lyon, \\ France \\ 4 Université Paris-Saclay, UVSQ, Univ. Paris-Sud, Inserm, CESP, Anti-infective evasion and pharma- coepidemiology team, Montigny-Le-Bretonneux, France \\ 5 Institut Pasteur, Epidemiology and Modelling of Evasion to Antibiotics, Paris, France \\ Corresponding Author: Matthieu Domenech de Cellès \\ Email address: domenech@mpiib-berlin.mpg.de
}

As in past pandemics, co-circulating pathogens may play a role in the epidemiology of coronavirus disease 2019 (COVID-19), caused by the novel severe acute respiratory syndrome coronavirus 2 (SARS-CoV-2). In particular, experimental evidence indicates that influenza infection can up-regulate the expression of ACE2-the receptor of SARS-CoV-2 in human cells-and facilitate SARS-CoV-2 infection. Here we hypothesized that influenza impacted the epidemiology of SARS-CoV-2 during the early 2020 epidemic of COVID-19 in Europe. To test this hypothesis, we developed a population-based model of SARS-CoV-2 transmission and of COVID-19 mortality, which simultaneously incorporated the impact of non-pharmaceutical control measures and of influenza on the epidemiological dynamics of SARS-CoV-2. Using statistical inference methods based on iterated filtering, we confronted this model with mortality incidence data in four European countries (Belgium, Italy, Norway, and Spain) to systematically test a range of assumptions about the impact of influenza. We found consistent evidence for a 1.8-3.4-fold (uncertainty range across countries: 1.1 to 5.0) average population-level increase in SARS-CoV-2 transmission associated with influenza during the period of co-circulation. These estimates remained robust to a variety of alternative assumptions regarding the epidemiological traits of SARSCoV-2 and the modeled impact of control measures. Although further confirmatory evidence is required, our results suggest that influenza could facilitate the spread and hamper effective control of SARS-CoV-2. More generally, our results highlight the possible role of co-circulating pathogens in the epidemiology of COVID-19. 
1 Estimating the impact of influenza on the epidemiological dynamics of SARS-CoV-2

2

3

4 1. Max Planck Institute for Infection Biology, Infectious Disease Epidemiology group,

$5 \quad$ Charitéplatz 1, Campus Charité Mitte, 10117 Berlin, Germany

6 2. Laboratoire de Virologie des HCL, IAI, CNR des virus à transmission respiratoire (dont la

7 grippe) Hôpital de la Croix-Rousse F-69317 Lyon cedex 04, France

8 3. Virpath, Centre International de Recherche en Infectiologie (CIRI), Université de Lyon

9 Inserm U1111, CNRS UMR 5308, ENS de Lyon, UCBL F-69372 Lyon cedex 08, France

10

*Corresponding author:

4. Université Paris-Saclay, UVSQ, Univ. Paris-Sud, Inserm, CESP, Anti-infective evasion and pharmacoepidemiology team, F-78180 Montigny-Le-Bretonneux, France

5. Institut Pasteur, Epidemiology and Modelling of Evasion to Antibiotics F-75015 Paris, France

Dr. Matthieu Domenech de Cellès, Max Planck Institute for Infection Biology, Charitéplatz 1,

16 Campus Charité Mitte, 10117 Berlin, Germany. E-mail address: domenech@mpiib-

17 berlin.mpg.de

Keywords

SARS-CoV-2; COVID-19; influenza; virus-virus interaction; mathematical modeling 
21 As in past pandemics, co-circulating pathogens may play a role in the epidemiology of 22 coronavirus disease 2019 (COVID-19), caused by the novel severe acute respiratory syndrome 23 coronavirus 2 (SARS-CoV-2). In particular, experimental evidence indicates that influenza 24 infection can up-regulate the expression of ACE2 - the receptor of SARS-CoV-2 in human 25 cells - and facilitate SARS-CoV-2 infection. Here we hypothesized that influenza impacted the 26 epidemiology of SARS-CoV-2 during the early 2020 epidemic of COVID-19 in Europe. To test 27 this hypothesis, we developed a population-based model of SARS-CoV-2 transmission and of 28 COVID-19 mortality, which simultaneously incorporated the impact of non-pharmaceutical 29 control measures and of influenza on the epidemiological dynamics of SARS-CoV-2. Using statistical inference methods based on iterated filtering, we confronted this model with mortality

31 incidence data in four European countries (Belgium, Italy, Norway, and Spain) to systematically 32 test a range of assumptions about the impact of influenza. We found consistent evidence for a 1.8-3.4-fold (uncertainty range across countries: 1.1 to 5.0 ) average population-level increase in 34 SARS-CoV-2 transmission associated with influenza during the period of co-circulation. These estimates remained robust to a variety of alternative assumptions regarding the epidemiological traits of SARS-CoV-2 and the modeled impact of control measures. Although further confirmatory evidence is required, our results suggest that influenza could facilitate the spread and hamper effective control of SARS-CoV-2. More generally, our results highlight the possible role of co-circulating pathogens in the epidemiology of COVID-19. 
40

41 The current pandemic of coronavirus disease 2019 (COVID-19), caused by the novel severe

42 acute respiratory syndrome coronavirus 2 (SARS-CoV-2), has led to global alarm. Following the

43 first case reports in December 2019 in Wuhan, China [1], SARS-CoV-2 rapidly spread across the

44 globe and has resulted in approximately 171 million cases and 3.6 million deaths worldwide, as

45 of June 1, 2021 [2]. Because of the initial lack of prophylactic or therapeutic treatments, the

46 pandemic caused the implementation of unprecedented control measures, which culminated in

47 the lockdown of several billion people in over 100 countries during April-May 2020 [3]. Although a number of fixed (e.g., greater age, male sex) and chronic (e.g., hypertension, diabetes) risk factors of mortality have now been identified [4], the time-varying drivers of COVID-19 epidemiology remain poorly understood. Experience gained from past pandemics has highlighted the potentially large contribution of co-circulating pathogens to the burden of an emerging disease [5]. Despite the relevance for epidemic forecasting and for designing control strategies, the impact of co-circulating pathogens on SARS-CoV-2 epidemiology has remained largely unexplored [6].

Respiratory viruses - including SARS-CoV-2 and other coronaviruses, rhinoviruses, influenza viruses, etc.-form a large class of viruses that cause seasonal infections of the respiratory tract in humans. Mounting evidence indicates that their epidemiologies are not independent, as a result of interaction mechanisms that may operate at different scales and that can be classified as either facilitatory or antagonistic [7,8]. The interaction between the respiratory syncytial virus (RSV) and influenza may provide an example of antagonism. Indeed, experimental evidence in ferrets has shown that influenza viruses induce an antiviral state that transiently limits secondary infection with RSV [9], an effect postulated to explain the delayed 
63 epidemic of RSV during the 2009 influenza pandemic[10,11]. Although such antagonistic

64 interactions appear, to date, to be the most common among respiratory viruses [8], experimental

65 evidence indicates that co-infections may have a facilitatory effect, for example by increasing

66 viral growth [12]. Increased transmission of influenza during co-infection with other respiratory

67 viruses was also proposed to explain the multiple waves during the 1918 influenza

68 pandemic [13]. Interestingly, according to recent evidence a viral respiratory infection (in

69 particular with influenza viruses) can up-regulate the expression of ACE2 - the cognate receptor

70 of SARS-CoV-2 in human cells - in the respiratory epithelium [14,15]. In addition, this up-

71 regulation was demonstrated experimentally to increase infectivity of SARS-CoV-2 in mice co-

72 infected with influenza A virus [16]. This suggests that respiratory viruses could affect the

73 epidemiology of SARS-CoV-2. Here, we hypothesized that influenza-which peaked in

74 February 2020 and therefore co-circulated during the early spread of COVID-19 in Europe

75 (Fig. 1B) - interacted with SARS-CoV-2.

To test this hypothesis, we developed a semi-mechanistic, population-based model of

77 SARS-CoV-2 transmission and of COVID-19 mortality. Using likelihood-based statistical

78 inference methods, we confronted this model to mortality incidence data in four European

79 countries to test a range of assumptions about the impact of influenza on the transmission

80 dynamics of SARS-CoV-2. We find that influenza may have transiently increased the

81 transmission of SARS-CoV-2 during the first wave of COVID-19 in Europe. Although further

82 confirmatory evidence is required, our results suggest that influenza could facilitate the spread of

83 SARS-CoV-2 and, more generally, emphasize the potential role of co-circulating pathogens in

84 the epidemiology of COVID-19. 
85

86

87

88

89

90

91

93

94

95

96

97

\section{Influenza incidence data}

103 Virological data on the weekly numbers of samples tested and of samples positive to any

104 influenza virus were available from the FluNet database, compiled by the WHO (Fig. S1A).

105 Parallel syndromic data on the weekly incidence rate of influenza-like illnesses (ILI) were 106 available from the FluID database, also compiled by the WHO(Fig. S1B). These data were 
107 deemed high-quality and used in a previous study on influenza forecasting in the countries

108 considered here [18]. The weekly incidence rate of influenza was then calculated as the product

109 of ILI incidence and of the fraction of samples positive to any influenza virus (Fig. 1B). Because

110 the magnitude of influenza incidence thus calculated varied markedly across countries (e.g., as a

111 result of different surveillance systems and case definitions), we rescaled each time series by its

112 average during the period of co-circulation of influenza and SARS-CoV-2 (Fig. 1B). The

113 resulting time series was therefore dimensionless and equalled 1 when influenza incidence

114 equalled its average value during that period.

\section{COVID-19 mortality data}

116 Data on the daily number of deaths caused by SARS-CoV-2 (counted by date of death) were 117 available from national public health public institutes, in Belgium (Sciensano [19]), in Italy 118 (Dipartimento della Protezione Civile [20]), and in Spain (Instituto de Salud Carlos III, official 119 data with historical corrections compiled by the media DATADISTA [21]). In Norway, the data 120 were available from the worldwide database compiled by the European Center for Disease 121 Control and Prevention [22]. Following a previous study [17], and to avoid a possible bias caused

122 by the dominance of deaths due to non-locally acquired infections early in the epidemic, we 123 included observed deaths from the date after which the cumulative observed death count 124 exceeded 10. Data points before that date were treated as missing and were assigned a 125 conditional log-likelihood of 0 , such that they did not contribute to the overall log-likelihood.

126 The data were not further pre-processed, except in Italy, where a negative death count was 127 reported on 24 June 2020 and was treated as missing and also assigned a log-likelihood of 0. 
128

129

130

131

133

135

136

137

138

139

140

141

\section{Transmission model}

\section{Model formulation}

We formulated a variant of the standard Susceptible-Exposed-Infected-Recovered transmission model [23], using the method of stages to allow for a realistic distribution of the latent, infectious, and onset-to-death periods [24,25]. Specifically, we assumed that the latent and infectious periods were Erlang-distributed with shape parameter 2 and mean $1 / \sigma=4$ days and $1 / \gamma=5$ days, respectively [26]. The resulting generation time $T_{g}$ (i.e., the time from infection of a primary case to transmission to a secondary case) had a mean of 6.5 days and a coefficient of variation of 0.58 (see Fig. S2 for the full distribution and the details of the calculation), consistent with empirical observations and with the values fixed in a previous modeling study [17,27]. To model the impact of the gradual implementation of non-pharmaceutical control measures (e.g., border closure, school closure, lockdown), we mapped the stringency index (denoted by $s i(t)$ ) to the time-varying relative reduction in transmission of SARS-CoV-2(denoted by $r_{\beta}(t)$ ). Specifically, we used the following simple linear scaling function, with saturation:

$$
r_{\beta}(t)=\min \left(1, b \times \frac{s i(t)}{100}\right)
$$

143 Here the parameter $b$ quantifies how steeply the transmission rate of SARS-CoV-2 decreases as

144 the stringency index increases. Hence, this parameter can be interpreted as a measure of the 145 impact of non-pharmaceutical control measures on SARS-CoV-2 transmission. The deterministic 146 variant of the model was represented by the following set of differential equations: 


$$
\begin{array}{ccc}
\dot{S} & = & -\lambda(t) S \\
\dot{E}_{1} & = & \lambda(t) S-2 \sigma E_{1} \\
\dot{E}_{2} & = & 2 \sigma\left(E_{1}-E_{2}\right) \\
\dot{I}_{1} & = & 2 \sigma E_{2}-2 \gamma I_{1} \\
\dot{I}_{2} & = & 2 \gamma\left(I_{1}-I_{2}\right) \\
\dot{R} & = & 2 \gamma I_{2}
\end{array}
$$

148 The force of infection (that is, the per capita rate at which susceptible individuals contract

149 infection [23]), $\lambda(t)$, was modeled as:

150

$$
\begin{array}{rlc}
\lambda(t) & = & \beta(t) \frac{I_{1}+I_{2}}{N} \\
\beta(t) & = & \beta_{0}\left(1-r_{\beta}(t)\right) \beta_{F}(t) \\
r_{\beta}(t) & = & \min \left(1, b \times \frac{\operatorname{si}(t)}{100}\right) \\
\beta_{F}(t) & = & \max \left(0,1+\beta_{F} F(t)\right) \\
R_{e}(t) & = & \frac{\beta(t)}{\gamma} \times \frac{S(t)}{N}
\end{array}
$$

151 where $R_{0}$ represents the basic reproduction number of SARS-CoV-2, $\beta_{0}=R_{0} \gamma$ the basic

152 transmission rate, $R_{e}(t)$ the time-varying effective reproduction number, $N$ the population size

153 (assumed constant during the study period), and $F(t)$ the renormalized time series of influenza

154 incidence, incorporated as a covariate into the model (Fig. 1). With this formulation, the

155 parameter $\beta_{F}$ quantifies the impact of influenza on SARS-CoV-2 transmission: $\beta_{F}>0$ if

156 influenza increases transmission, $\beta_{F}<0$ if influenza decreases transmission, and $\beta_{F}=0$ if

157 influenza has no impact on transmission (null hypothesis). More specifically, the average

158 incidence of influenza during the period of co-circulation with SARS-CoV-2 corresponds to

$159 F(t)=1$, such that $1+\beta_{F}$ represents the average relative variation of SARS-CoV-

1602 transmission associated with influenza. In writing the equations, we implicitly assume that the 
161 impact of influenza on SARS-CoV-2 transmission, if any, is short-lived and does not extend long

162 after influenza infection.

163

Finally, we incorporated an observation model that related the dynamics of SARS-CoV-

1642 infection to that of COVID-19 mortality, taking into account the fact that only a fraction of

165 infections results in death and that, among those, death occurs some time after symptom

166 onset [17,28,29]. We assumed an average duration of pre-symptomatic of 2.5 days, resulting in

167 an average incubation period of 6.5 days, in broad agreement with previous empirical

168 studies $[29,30]$. Hence, individuals in the first infected state $\left(I_{1}\right)$ were considered pre-

169 symptomatic, and the onset of symptoms was assumed to coincide with the transition from $I_{1}$ to

$170 I_{2}$. The onset-to-death time was then assumed to be Erlang distributed with shape parameter 5

171 and mean $1 / \kappa=17.8$ days (coefficient of variation of 0.45 ), the value estimated in a previous

172 epidemiological study [28]. In a sensitivity analysis, we also tested a mean onset-to-death time of

$1731 / \kappa=13$ days, the lower bound estimated in a meta-analysis [29]. According to previous studies

174 in European countries, the infection fatality ratio (IFR) typically ranged from $0.5 \%$ to $1 \%$ early

175 in the epidemic [31-33]. We fixed the IFR to $\mu=0.01$ in the base model, but we considered an

176 alternative value of 0.005 in a sensitivity analysis. Given those assumptions, the observation

177 model was modeled by the following set of ordinary differential equations:

178

$$
\begin{array}{ccc}
\dot{Q}_{1} & = & 2 \gamma \mu I_{1}-5 \kappa Q_{1} \\
\dot{Q}_{i=2, \ldots, 5} & = & 5 \kappa\left(Q_{i-1}-Q_{i}\right) \\
\dot{D}_{M} & = & 5 \kappa Q_{5}
\end{array}
$$

179 Here $D_{M}$ is the simulated number of daily deaths, modeled as an accumulator variable and reset 180 to 0 at the end of each day. The observed number of daily deaths, $D_{O}$, was modeled using a 
181 negative binomial distribution with mean $D_{M}$ and over-dispersion $k_{D}$ (i.e., $\mathbb{V}\left(D_{O} \mid D_{M}\right)=D_{M}+k_{D}$

$182 D_{M}^{2}$ ), a standard distribution used in previous modeling studies [17,34].

183

As in Flaxman et al. [17], simulations were started 30 days before the date from which

184 the cumulative observed death count first exceeded 10. At that date, we assumed that $E_{1}(0)$

185 individuals had been exposed to SARS-CoV-2; other individuals were assumed susceptible to

186 infection (i.e., $S(0)=N-E_{1}(0)$ ), and all other compartments were initialized to 0 .

\section{Stochastic variant and modeling of superspreading}

188 The stochastic variant of the model was implemented as a continuous-time Markov process 189 approximated via a multinomial modification of the $\tau$-leap algorithm [35], with a fixed time step

190 of $\Delta t=10^{-1}$ day. To model the effect of superspreading events - a key feature of SARS-CoV-

1912 transmission dynamics [36] - extra-demographic stochasticity was added to the transmission

192 rate $\beta(t)$. Specifically, as proposed by Kain et al. [37], at every time step we drew a value of $\beta_{0}$

193 from a Gamma white noise distribution:

194

$$
\beta_{0} \sim \Gamma_{\mathrm{WN}}\left(\sigma=\sqrt{\frac{R_{0}}{\left(I_{1}+I_{2}\right) k \Delta t}} \mu=R_{0} \gamma\right)
$$

195 with mean $\mu$ and variance $\mu \sigma^{2}$. Here $k$ represents the dispersion parameter of the Negative-

196 binomial distribution for the individual reproduction number (with mean $R_{0}$ and variance $R_{0}+\frac{R_{0}^{2}}{k}$

197 ), as estimated in previous studies [38,39]. As in [37] and in keeping with empirical estimates 198 from contact tracing studies of SARS-CoV-2 [39], we fixed $k=0.16$. 
200 Following the method presented in[17], we estimated unknown model parameters using observed

201 COVID-19 mortality data alone. Indeed, because of the initially limited testing capacity 202 (typically reserved to severe cases or high-risk groups), mortality data were arguably more 203 reliable than case data early in the epidemic in most countries [17]. By incorporating known 204 epidemiological parameters (key among those the onset-to-death time, the IFR, and the 205 generation time), however, the method allows back-calculating infection rates from observed 206 death rates. Hence, in addition to the dynamic of mortality, we also reconstructed the dynamic of 207 infection and, as a validation, compared it to external epidemiological data-like cross-sectional 208 seroprevalence estimates — when available.

The following five parameters were estimated from the data:

210 1. The basic reproduction number, $R_{0}$. According to a previous meta-analysis [40], this 211 parameter was searched in the interval 1-10.

212 2. The impact of non-pharmaceutical control measures, $b$. The lower bound of the search 213 interval of this parameter was fixed to 0.5 , such that the maximal value of the stringency 214 index $(s=100)$ corresponded to a minimal reduction of SARS-CoV-2 transmission of $215 \quad 50 \%[17]$.

216 3. The impact of influenza on SARS-CoV-2 transmission, $\beta_{F}$ (search interval: $\mathbb{R}$ ).

217 4. The initial number of individuals exposed to SARS-CoV-2, $E_{1}(0)$ (search interval: $\left.0-10^{4}\right)$.

218 5. The over-dispersion in death reporting, $k_{D}\left(\right.$ search interval: $\left.\mathbb{R}^{+}\right)$. 
219 A summary list of fixed and estimated model parameters is presented in Table 1.

220

All parameters were transformed to be estimated on the real line, using a log

221 transformation for positive parameters and the extended logistic function $f(\theta)=\log _{b-\theta} \frac{\theta-a}{b o r}$

222 parameters constrained in the interval $[a, b]$. The maximum iterated filtering algorithm

223 (MIF2 [41]), implemented in the R (version 3.6.3) package pomp [42,43] (version 2.7), was used

224 to estimate model parameters. The R checkpoint package was used to freeze all the packages'

225 version at the date of April 3, 2020 [44]. The estimation was completed in several steps, starting

226 with trajectory matching to identify good starting parameters for MIF2, followed by 100

227 independent runs of MIF2 to locate the maximum likelihood estimate (MLE). Each MIF run had

228150 iterations with 5,000 particles, geometric cooling, and a random walk standard deviation of

2290.1 for the initial condition $E_{1}(0)$ and of 0.02 for the other parameters. The log-likelihood of

230 every parameter set was calculated as the log of the mean likelihood of 5 replicate particle filters,

231 each with 20,000 particles. The profile likelihood was calculated to verify the convergence of

232 MIF2 and to derive an approximate $95 \%$ confidence interval for the parameter $\beta_{F}[45]$ - the

233 parameter of key interest in our study. For the other parameters, a parametric bootstrap was used

234 to calculate approximate $95 \%$ confidence intervals, by re-estimating the parameters for each of

235200 synthetic datasets simulated at the MLE [46,47]. Compared with the profile likelihood, the

236 parametric bootstrap requires less computation and was found to perform well in previous

237 applications $[46,47]$.

238 


\section{Sensitivity analyses}

240 To verify the robustness of the parameter estimates, we conducted six sensitivity analyses

241 (further detailed in the Supplementary Results). First, we estimated an extended model in which

242 the reduction of SARS-CoV-2 transmission was allowed to scale non-linearly with the stringency

243 index. Second, to test the possible presence of other variables confounded with influenza, we

244 estimated a model that included an exponential trend in the transmission rate of SARS-CoV-2.

245 Finally, we varied the fixed value of 3 parameters and re-estimated the parameters of the base

246 model. Specifically, we tested two alternative values of the average generation time $\left(\mathbb{E}\left(T_{g}\right)=5\right.$

247 days and $\mathbb{E}\left(T_{g}\right)=7.5$ days $)$, one alternative value of the infection fatality ratio $(\mu=0.005)$, and

248 one alternative value of the average onset-to-death period $\left(\frac{1}{\kappa}=13\right.$ days $)$.

249 Results

250 Parameter estimates

251 As shown in Fig. 1A, the number and the intensity of control measures against COVID-

25219 gradually increased from January until the nationwide lockdown in March, before a relaxation

253 from May 2020. During this time period, the epidemic of influenza started in January 2020 and

254 ended in March 2020, with a peak during February in each country (Fig. 1B and S1). Despite

255 correlations between some parameters (in particular the reproduction number and the impact of

256 control measures, see Fig. S4), all parameters were identifiable in each country (Table 2).

257 Parameter estimates indicated that, during the period of co-circulation, influenza was associated

258 with an average 1.8-3.4-fold (uncertainty range across countries: 1.1 to 5.0) population-level

259 increase in SARS-CoV-2 transmission (Table 2 and Fig. S3). After controlling for the impact of

260 influenza, our estimates of the basic reproduction number $\left(R_{0}\right)$ ranged from 1.2 (in Italy) to 3.4 
261 (in Belgium). Although the increased transmission associated with influenza early during the

262 SARS-CoV-2 epidemic explained the data significantly better (Table 2), a model without

263 influenza led to higher $R_{0}$ estimates (range 2.4-5.2, Fig. 2A), consistent with those of a previous

264 study [17]. Also in line with [17], we found consistent evidence for a marked impact of non-

265 pharmaceutical control measures (Table 2), which were associated with a decrease in SARS-

266 CoV-2 transmission below the reproduction threshold from mid-March to June 2020 (Fig. 2A).

267 Model evaluation

268 Visual inspection of simulations suggested that our model correctly captured the dynamics of

269 COVID-19 mortality in every country (Fig. 2B). A more detailed model-data comparison of

270 summary statistics confirmed that our model accurately reproduced the peak time, the peak

271 number and the total number of deaths, and the death growth exponent [48], except in Italy and

272 Spain where the latter statistic was systematically under-estimated (Fig. S5). Our model-based

273 estimates of the total proportion of individuals infected with SARS-CoV-2 (as of 4 May 2020,

274 Table 2) were also comparable with those of a previous modeling study [17] and of a

275 seroprevalence study conducted in early May in Spain (approximate seroprevalence estimate of

$2765 \%$ [49]). Hence, our model appeared to precisely recapitulate the epidemiology of SARS-CoV-

2772 morbidity and mortality over a period of $\sim 4$ months.

278 Sensitivity analyses

279 The results of the sensitivity analyses are presented in Tables S1-S3. We found little statistical

280 evidence that the model with non-linear scaling of the stringency index outperformed the model

281 with simple linear scaling in any country $(\Delta \log L \in[0.0,1.1]$, likelihood ratio test P-value $282 P \in[0.14,1.00]$, Table S1). Of note, although our estimates of the impact of influenza varied 
283 little, the additional estimated parameter resulted in higher parametric uncertainty, particularly in

284 Norway where the approximate 95\% CI embraced the null value. Similarly, the model with an

285 unexplained exponential trend in transmission did not substantially improve model fit $(\Delta \log$

$286 L \in[0.0,3.2]$, likelihood ratio test P-value $P \in[0.01,1.00]$, Table S2). Despite higher parametric

287 uncertainty caused by the estimation of the trend, our results regarding the impact of influenza

288 remained robust, but once again the approximate confidence interval embraced the null value in

289 Norway. In addition, we found that the parameter estimates varied little when testing alternative

290 hypotheses about the fixed value of the average generation time, of the onset-to-death time, and

291 of the infection fatality ratio (Table S3). Finally, because our $R_{0}$ estimates were lower than

292 previous estimates in Italy and Spain, we tested an alternative model with $R_{0}$ fixed to 2.5 in each

293 country. This model led to broadly similar conclusions, although the estimated impact of

294 influenza was lower both in Italy $\left(\beta_{F}=1.4\right.$, approximate 95\% CI $[1.2,1.5], \log L=-692.3$

$295[\mathrm{SE}<0.1])$ and in Spain $\left(\beta_{F}=0.7\right.$, approximate 95\% CI $[0.4,1.0], \log L=-567.7$ [SE $\left.\left.=0.1\right]\right)$. In

296 sum, our main result about the impact of influenza remained robust to a variety of alternative

297 assumptions regarding the epidemiological traits of SARS-CoV-2 and the modeled impact of

298 control measures.

299 Discussion

300 The main goal of this study was to test the hypothesis that influenza impacted the 301 epidemiological dynamics of SARS-CoV-2, building on previous experimental evidence of a 302 positive interaction between the two viruses[14-16]. To do so, we developed a semi303 mechanistic, population-based model of SARS-CoV-2 transmission and of COVID-19 mortality, 304 which simultaneously incorporated the impact of non-pharmaceutical control measures and of 
305 influenza. Using likelihood-based statistical inference techniques, we confronted this model with

306 mortality incidence data in four European countries to systematically test a range of assumptions

307 about the possible impact of influenza and of control measures. In keeping with previous

308 studies [17], we found robust and consistent evidence that control measures markedly reduced

309 the transmission of SARS-CoV-2. In addition, we also found consistent evidence suggesting that

310 co-circulation of influenza transiently facilitated the transmission of SARS-CoV-2 early in the

311 epidemic in Europe.

Our study has a number of important limitations. First, as in other studies $[17,26,50]$ and

313 because of a lack of appropriate age-specific data (for example on the temporal changes in the

314 contact matrix and in the incidence of influenza), our model was not age-structured, even though

315 many aspects of COVID-19 and of influenza epidemiology-like disease severity and

316 lethality—vary markedly with age [28]. The susceptibility to SARS-CoV-2 infection was also

317 found to increase with age [51], a finding potentially explained by lower baseline expression of

318 the ACE2 receptor in children[52]. A testable prediction of our model, therefore, is that

319 influenza should be associated with a transient increase in susceptibility to SARS-CoV-

3202 infection, commensurate with the variations of influenza incidence over age. Second, we

321 modeled the impact of non-pharmaceutical control measures using a simple, linear function

322 scaling the stringency index to the reduction of SARS-CoV-2 transmission. Even though this

323 simple hypothesis provided a more parsimonious fit (except in Italy), that result may be specific

324 to Europe, where control measures gradually increased in number and in intensity (Fig. 1A). In

325 general, the association is likely non-linear (e.g., if a high-impact intervention like a lockdown is

326 implemented early on), and we therefore recommend testing a variety of scaling functions. More

327 generally, although our model builds on a previously validated method to estimate the time- 
328 varying reproduction number[17], we acknowledge that the stringency index may not fully

329 capture temporal variations in SARS-CoV-2 transmission, in particular behavior changes outside

330 of what was mandated by governments. Even though we tested a model with an unexplained

331 trend in transmission (Table S2), more complex temporal functions may be required to fully

332 capture such changes. Third, we did not incorporate climate into our model, even though, as for

333 other respiratory viruses, environmental variables like temperature and humidity may affect the

334 transmission of SARS-CoV-2. According to previous studies conducted in a variety of locations

335 worldwide, however, the impact of weather on the SARS-CoV-2 epidemic appears to have been

336 modest, at least during the first wave in early 2020 [53-55]. These findings are also consistent

337 with epidemiological theory, which predicts that, because of lack of population immunity, the

338 initial pandemic trajectory may be relatively insensitive to climate [56]. Fourth, we did not

339 specifically model fully asymptomatic cases, which may represent a large fraction of SARS-

340 CoV-2 infections [26]. The omission of asymptomatic infections may lead to biased $R_{0}$ estimates

341 if their duration significantly differs from that of symptomatic infections [57]. A previous study,

342 however, estimated that the duration of both types of infection is comparable [26], such that our

343 estimates should be robust in more complex model structures. Finally, we assessed only the

344 impact of influenza, because of its high prevalence and period of overlap with SARS-CoV-2 in

345 early 2020 in Europe and of the availability of high-quality data [18]. Nevertheless, other

346 respiratory viruses, like RSV and rhinoviruses [58], may also interact with SARS-CoV-2 and

347 could be considered.

348 Acknowledging these limitations, our model makes at least two other predictions that

349 could be tested to provide confirmatory evidence. First, even though our results did not allow to

350 distinguish between higher transmissibility or higher susceptibility in individuals co-infected 
351 with influenza and SARS-CoV-2, previous experimental work suggests that the latter mechanism

352 may operate, as a result of up-regulation of the ACE2 receptor caused by influenza

353 infection $[14,15]$. Hence, we predict that a recent influenza infection should be an independent

354 risk factor for subsequent SARS-CoV-2 infection. Estimates of the frequency of co-detection of 355 influenza and SARS-CoV-2 by polymerase chain reaction (PCR) testing in nasopharyngeal 356 swabs were highly variable in previous studies (range $0-60 \%[6,59]$ ). Although the marked 357 seasonality of influenza in temperate regions likely explains in part the low frequency found in 358 some studies [59], we propose that differences in the natural history of influenza and SARS359 CoV-2 infections also lead to a systematic under-estimation of co-infection. Specifically, because 360 the incubation period of SARS-CoV-2 infection (estimated to average 5.7 days [29]) exceeds that 361 of influenza (A, 1.4 days or B, 0.6 days [60]), it is likely that, by the time SARS-CoV-2 infection 362 becomes detectable, influenza no longer is. To make that statement more precise, we calculated 363 the probability of detectability of a co-infection, with influenza first then SARS-CoV3642 (Table S4). Assuming that influenza is detectable by PCR up to 4-5 days after [61], and SARS365 CoV-2 from 2-4 days before [30], symptom onset, we find that a large fraction (30-50\%) of co366 infections may not be detectable at all. These results may help explain the low frequency of co367 detection found in some studies [62], and suggest that the time window of co-detectability may 368 be too short to adequately infer the association between influenza and SARS-CoV-2 using PCR 369 testing. Serological studies comparing the prevalence of antibodies against influenza in SARS$370 \mathrm{CoV}-2$ cases and non-cases may therefore be required to test the prediction that influenza is a 371 risk factor for SARS-CoV-2 infection. Second, we predict that individuals vaccinated against 372 influenza should be at lower risk of SARS-CoV-2 infection than those unvaccinated. The 373 findings of a negative association between influenza vaccine coverage and COVID-19 mortality 
374 in ecological studies (in Italy [63] and in other countries [64]) and of a lower risk of SARS-CoV-

3752 infection in influenza vaccinees in some individual-level epidemiological studies (reviewed

376 in [65]) are consistent with our prediction, but further epidemiological investigations are needed.

377 Importantly, our results can explain these findings as the direct effect of influenza vaccines on

378 influenza infection, instead of indirect effects on non-influenza pathogens (e.g., as a result of

379 trained immunity) [66].

With the likely prospect of COVID-19 becoming endemic, the potential interactions of

381 SARS-CoV-2 with other respiratory pathogens - in particular respiratory viruses - may become

382 a key public health issue. In this context, our results suggest that influenza could facilitate the

383 circulation of SARS-CoV-2 and therefore increase the burden of COVID-19. As outlined above,

384 these results are consistent with several lines of experimental $[16,14,15]$ and epidemiological

$385[65,64,63]$ evidence. We note, however, that a previous study proposed that influenza and 386 SARS-CoV-2 have competitive interactions [67]. Specifically, using a within- host model of 387 viral replication Pinky and Dobrovolny found that the low growth rate of SARS-CoV-2 may 388 result in limited access to target cells and therefore suppression by other respiratory viruses [67]. 389 Although this mechanism may be generally relevant for respiratory viruses, the ability of 390 influenza to up-regulate ACE2 $[14,15]$ —a feature not included in the within-host model [67]— 391 could counteract this mechanism and explain the increased infectivity of SARS-CoV-2 found 392 experimentally [16]. Of note, in keeping with [67], another experimental study found evidence 393 that the interferon response caused competitive interactions between rhinoviruses and SARS394 CoV-2 [58]. Hence, these different biological mechanisms suggest that every respiratory virus 395 interacts with SARS-CoV-2in a highly specific way, with influenza being unique in its ability to 396 up-regulate ACE2 and to increase SARS-CoV-2 infectivity [16]. 
399 In conclusion, our results suggest that influenza virus infection could have increased the 400 transmission of SARS-CoV-2 and facilitated its spread during the early 2020 epidemic of 401 COVID-19 in Europe. Hence, an increase in the uptake of influenza vaccines may be called for, 402 not only to reduce hospitalizations due to influenza infections [59,68], but also to reduce their 403 downstream impact on SARS-CoV-2 transmission and on COVID-19 mortality. More generally, 404 taking into account the microbial environment of SARS-CoV-2 may be essential, not only to 405 better understand its epidemiology, but also to enhance current and future infection control 406 strategies. 


\section{Acknowledgements}

\section{Availability of data and materials}

409 The data used for this analysis are freely available from the databases cited in the references and

410 have been compiled into a database available from Edmond, the Open Data Repository of the

411 Max Planck Society: https://edmond.mpdl.mpg.de/imeji/collection/eUiBI4vpiGxi4126. The R

412 programming codes to implement and estimate the models are also available on this repository.

\section{Competing interests}

414 MDdC received postdoctoral funding (2017-2019) from Pfizer and consulting fees from GSK.

415 JSC declares no competing interests. BL is chair of the ISC for the Global Influenza Surveillance

416 Network, and co-chair of the Global Influenza and RSV initiative; he is also a member of the

417 French COVID-19 Scientific Committee (no personal income for all these activities). LO has

418 received consulting fees from $\mathrm{WHO}$ for work on antimicrobial resistance and funding from

419 Pfizer (2017-2019).

420

Authors contributions

421 MDdC conceived of the study design and performed the analysis. JSC and BL provided content

422 expertise. LO conceived of the study design and oversaw the analysis.

\section{Acknowledgements}

424 We thank Arturo Zychlinsky and Klaus Osterrieder for helpful comments on the manuscript.

425 Computations underlying the present analysis were performed at the Max Planck Computing and 426 Data Facility (MPCDF). 
427 References

428

429 1. Zhu N, Zhang D, Wang W, Li X, Yang B, Song J, Zhao X, Huang B, Shi W, Lu R, Niu P, 430 Zhan F, Ma X, Wang D, Xu W, Wu G, Gao GF, Tan W, and China Novel Coronavirus

431 Investigating and Research Team. 2020. A Novel Coronavirus from Patients with Pneumonia in 432 China, 2019. N Engl J Med 382:727-733. 10.1056/NEJMoa2001017

433 2. Johns Hopkins University Center for Systems Science and Engineering (JHU CCSE). Novel 434 coronavirus (covid-19) cases data [Internet]. Available from:

435 https://data.humdata.org/dataset/novel-coronavirus-2019-ncov-cases

436 3. Hale T, Webster S, Petherick A, Phillips T, Kira B. Oxford covid-19 government response 437 tracker. Blavatnik School of Government Working Paper [Internet]. Available from:

438 www.bsg.ox.ac.uk/covidtracker

439 4. Williamson EJ, Walker AJ, Bhaskaran K, Bacon S, Bates C, Morton CE, Curtis HJ, Mehrkar

440 A, Evans D, Inglesby P, Cockburn J, McDonald HI, MacKenna B, Tomlinson L, Douglas IJ, 441 Rentsch CT, Mathur R, Wong AYS, Grieve R, Harrison D, Forbes H, Schultze A, Croker R, 442 Parry J, Hester F, Harper S, Perera R, Evans SJW, Smeeth L, and Goldacre B. 2020. Factors 443 associated with COVID-19-related death using OpenSAFELY. Nature. 10.1038/s41586-020$444 \quad 2521-4$

445 5. Morens DM, Taubenberger JK, and Fauci AS. 2008. Predominant role of bacterial pneumonia 446 as a cause of death in pandemic influenza: implications for pandemic influenza preparedness. $J$ 447 Infect Dis 198:962-970. 10.1086/591708 
448 6. Thindwa D, Garcia Quesada M, Liu Y, Bennett J, Cohen C, Knoll MD, von Gottberg A,

449 Hayford K, and Flasche S. 2020. Use of seasonal influenza and pneumococcal polysaccharide

450 vaccines in older adults to reduce COVID-19 mortality. Vaccine 38:5398-5401.

$451 \quad 10.1016 /$ j.vaccine.2020.06.047

452 7. DaPalma T, Doonan BP, Trager NM, and Kasman LM. 2010. A systematic approach to virus453 virus interactions. Virus Res 149:1-9. 10.1016/j.virusres.2010.01.002

454 8. Opatowski L, Baguelin M, and Eggo RM. 2018. Influenza interaction with cocirculating 455 pathogens and its impact on surveillance, pathogenesis, and epidemic profile: A key role for 456 mathematical modelling. PLoS Pathog 14:e1006770. 10.1371/journal.ppat.1006770

457 9. Chan KF, Carolan LA, Korenkov D, Druce J, McCaw J, Reading PC, Barr IG, and Laurie KL. 458 2018. Investigating Viral Interference Between Influenza A Virus and Human Respiratory 459 Syncytial Virus in a Ferret Model of Infection. J Infect Dis 218:406-417. 10.1093/infdis/jiy184

460 10. Casalegno JS, Ottmann M, Bouscambert-Duchamp M, Valette M, Morfin F, and Lina B. 461 2010. Impact of the 2009 influenza A(H1N1) pandemic wave on the pattern of hibernal 462 respiratory virus epidemics, France, 2009. Euro Surveill 15.

463 11. Mak GC, Wong AH, Ho WYY, and Lim W. 2012. The impact of pandemic influenza A 464 (H1N1) 2009 on the circulation of respiratory viruses 2009-2011. Influenza Other Respir Viruses 465 6:e6-10. 10.1111/j.1750-2659.2011.00323.x

466 12. Goto H, Ihira H, Morishita K, Tsuchiya M, Ohta K, Yumine N, Tsurudome M, and Nishio 467 M. 2016. Enhanced growth of influenza A virus by coinfection with human parainfluenza virus 468 type 2. Med Microbiol Immunol 205:209-218. 10.1007/s00430-015-0441-y 
469 13. Merler S, Poletti P, Ajelli M, Caprile B, and Manfredi P. 2008. Coinfection can trigger

470 multiple pandemic waves. $J$ Theor Biol 254:499-507. 10.1016/j.jtbi.2008.06.004

471 14. Smith JC, Sausville EL, Girish V, Yuan ML, Vasudevan A, John KM, and Sheltzer JM.

472 2020. Cigarette Smoke Exposure and Inflammatory Signaling Increase the Expression of the

473 SARS-CoV-2 Receptor ACE2 in the Respiratory Tract. Dev Cell 53:514-529.e513.

$474 \quad 10.1016 /$ j.devcel.2020.05.012

475 15. Ziegler CGK, Allon SJ, Nyquist SK, Mbano IM, Miao VN, Tzouanas CN, Cao Y, Yousif 476 AS, Bals J, Hauser BM, Feldman J, Muus C, Wadsworth n, Marc H, Kazer SW, Hughes TK, 477 Doran B, Gatter GJ, Vukovic M, Taliaferro F, Mead BE, Guo Z, Wang JP, Gras D, Plaisant M, 478 Ansari M, Angelidis I, Adler H, Sucre JMS, Taylor CJ, Lin B, Waghray A, Mitsialis V, Dwyer 479 DF, Buchheit KM, Boyce JA, Barrett NA, Laidlaw TM, Carroll SL, Colonna L, Tkachev V, 480 Peterson CW, Yu A, Zheng HB, Gideon HP, Winchell CG, Lin PL, Bingle CD, Snapper SB, 481 Kropski JA, Theis FJ, Schiller HB, Zaragosi L-E, Barbry P, Leslie A, Kiem H-P, Flynn JL, 482 Fortune SM, Berger B, Finberg RW, Kean LS, Garber M, Schmidt AG, Lingwood D, Shalek 483 AK, Ordovas-Montanes J, HCA Lung Biological Network. Electronic address: lung484 network@humancellatlas.org, and HCA Lung Biological Network. 2020. SARS-CoV-2 485 Receptor ACE2 Is an Interferon-Stimulated Gene in Human Airway Epithelial Cells and Is 486 Detected in Specific Cell Subsets across Tissues. Cell 181:1016-1035.e1019.

487 10.1016/j.cell.2020.04.035 L, Liu Q, Zheng Y, Niu D, Xiang M, Song K, Ye J, Zheng W, Tang Z, Tang M, Zhou Y, Shen C, 
490 Dai M, Zhou L, Chen Y, Yan H, Lan K, and Xu K. 2021. Coinfection with influenza A virus

491 enhances SARS-CoV-2 infectivity. Cell Res 31:395-403. 10.1038/s41422-021-00473-1

492 17. Flaxman S, Mishra S, Gandy A, Unwin HJT, Mellan TA, Coupland H, Whittaker C, Zhu H,

493 Berah T, Eaton JW, Monod M, Imperial College COVID-19 Response Team, Ghani AC,

494 Donnelly CA, Riley SM, Vollmer MAC, Ferguson NM, Okell LC, and Bhatt S. 2020. Estimating

495 the effects of non-pharmaceutical interventions on COVID-19 in Europe. Nature.

$496 \quad 10.1038 / \mathrm{s} 41586-020-2405-7$

497 18. Kramer SC, and Shaman J. 2019. Development and validation of influenza forecasting for 64

498 temperate and tropical countries. PLoS Comput Biol 15:e1006742. 10.1371/journal.pcbi.1006742

499 19. Belgian institute for health (sciensano), access date: 6 july 2020 [Internet]. Available from:

500 https://epistat.wiv-isp.be/Covid/

501 20. Dipartimento della Protezione Civile,. COVID-19 italia-monitoraggio della situazione, date

502 of access: 6 july 2020 [Internet]. Available from: https://github.com/pcm-dpc/COVID-

503 19/tree/master/dati-andamento-nazionale

504 21. DATADISTA. Date of access: 6 july 2020 [Internet]. Available from:

505 https://github.com/datadista/datasets/tree/master/COVID 19

506 22. European Centre for Disease Prevention and Control. Geographic distribution of covid-19

507 cases worldwide, access date: 6 july 2020 [Internet]. Available from:

508 https://www.ecdc.europa.eu/en/publications-data/download-todays-data-geographic-distribution509 covid-19-cases-worldwide 
510 23. Keeling MJ, Rohani P. Modeling infectious diseases in humans and animals [Internet].

511 Princeton: Princeton University Press; 2008. Available from:

512 http://www.loc.gov/catdir/toc/fy0805/2006939548.html

513 24. Lloyd AL. 2001. Destabilization of epidemic models with the inclusion of realistic

514 distributions of infectious periods. Proc Biol Sci 268:985-993. 10.1098/rspb.2001.1599

515 25. Wearing HJ, Rohani P, and Keeling MJ. 2005. Appropriate models for the management of 516 infectious diseases. PLoS Med 2:e174. 10.1371/journal.pmed.0020174

517 26. Li R, Pei S, Chen B, Song Y, Zhang T, Yang W, and Shaman J. 2020. Substantial

518 undocumented infection facilitates the rapid dissemination of novel coronavirus (SARS-CoV2).

519 Science. 10.1126/science.abb3221

520 27. Bi Q, Wu Y, Mei S, Ye C, Zou X, Zhang Z, Liu X, Wei L, Truelove SA, Zhang T, Gao W,

521 Cheng C, Tang X, Wu X, Wu Y, Sun B, Huang S, Sun Y, Zhang J, Ma T, Lessler J, and Feng T.

522 2020. Epidemiology and transmission of COVID-19 in 391 cases and 1286 of their close

523 contacts in Shenzhen, China: a retrospective cohort study. Lancet Infect Dis. 10.1016/S1473-

$5243099(20) 30287-5$

525 28. Verity R, Okell LC, Dorigatti I, Winskill P, Whittaker C, Imai N, Cuomo-Dannenburg G,

526 Thompson H, Walker PGT, Fu H, Dighe A, Griffin JT, Baguelin M, Bhatia S, Boonyasiri A,

527 Cori A, Cucunubá Z, FitzJohn R, Gaythorpe K, Green W, Hamlet A, Hinsley W, Laydon D, 528 Nedjati-Gilani G, Riley S, van Elsland S, Volz E, Wang H, Wang Y, Xi X, Donnelly CA, Ghani 529 AC, and Ferguson NM. 2020. Estimates of the severity of coronavirus disease 2019: a model530 based analysis. Lancet Infect Dis. 10.1016/S1473-3099(20)30243-7 
531 29. Khalili M, Karamouzian M, Nasiri N, Javadi S, Mirzazadeh A, and Sharifi H. 2020.

532 Epidemiological characteristics of COVID-19: a systematic review and meta-analysis. Epidemiol 533 Infect 148:e130. 10.1017/S0950268820001430

534 30. Tindale LC, Stockdale JE, Coombe M, Garlock ES, Lau WYV, Saraswat M, Zhang L, Chen 535 D, Wallinga J, and Colijn C. 2020. Evidence for transmission of COVID-19 prior to symptom 536 onset. Elife 9. 10.7554/eLife.57149

537 31. Salje H, Tran Kiem C, Lefrancq N, Courtejoie N, Bosetti P, Paireau J, Andronico A, Hozé N, 538 Richet J, Dubost C-L, Le Strat Y, Lessler J, Levy-Bruhl D, Fontanet A, Opatowski L, Boelle P539 Y, and Cauchemez S. 2020. Estimating the burden of SARS-CoV-2 in France. Science.

$540 \quad 10.1126 /$ science.abc3517

541 32. O'Driscoll M, Ribeiro Dos Santos G, Wang L, Cummings DAT, Azman AS, Paireau J, 542 Fontanet A, Cauchemez S, and Salje H. 2021. Age-specific mortality and immunity patterns of 543 SARS-CoV-2. Nature 590:140-145. 10.1038/s41586-020-2918-0

544 33. Pastor-Barriuso R, Pérez-Gómez B, Hernán MA, Pérez-Olmeda M, Yotti R, Oteo-Iglesias J, 545 Sanmartín JL, León-Gómez I, Fernández-García A, Fernández-Navarro P, Cruz I, Martín M, 546 Delgado-Sanz C, Fernández de Larrea N, León Paniagua J, Muñoz-Montalvo JF, Blanco F, 547 Larrauri A, Pollán M, and ENE-COVID Study Group. 2020. Infection fatality risk for SARS548 CoV-2 in community dwelling population of Spain: nationwide seroepidemiological study. $B M J$ 549 371:m4509. 10.1136/bmj.m4509

550 34. King AA, Domenech de Cellès M, Magpantay FMG, and Rohani P. 2015. Avoidable errors 551 in the modelling of outbreaks of emerging pathogens, with special reference to Ebola. Proc Biol 552 Sci 282:20150347. 10.1098/rspb.2015.0347 
553 35. He D, Ionides EL, and King AA. 2010. Plug-and-play inference for disease dynamics:

554 measles in large and small populations as a case study. $J R$ Soc Interface 7:271-283.

$555 \quad 10.1098 /$ rsif.2009.0151

556 36. Althouse BM, Wenger EA, Miller JC, Scarpino SV, Allard A, Hébert-Dufresne L, and Hu H. 557 2020. Superspreading events in the transmission dynamics of SARS-CoV-2: Opportunities for 558 interventions and control. PLoS Biol 18:e3000897. 10.1371/journal.pbio.3000897

559 37. Kain MP, Childs ML, Becker AD, and Mordecai EA. 2020. Chopping the tail: How 560 preventing superspreading can help to maintain COVID-19 control. Epidemics 34:100430.

561 10.1016/j.epidem.2020.100430

562 38. Lloyd-Smith JO, Schreiber SJ, Kopp PE, and Getz WM. 2005. Superspreading and the effect 563 of individual variation on disease emergence. Nature 438:355-359. 10.1038/nature04153

564 39. Endo A, Centre for the Mathematical Modelling of Infectious Diseases COVID-19 Working 565 Group, Abbott S, Kucharski AJ, and Funk S. 2020. Estimating the overdispersion in COVID-19 566 transmission using outbreak sizes outside China. Wellcome Open Res 5:67.

567 10.12688/wellcomeopenres. 15842.3

568 40. Alimohamadi Y, Taghdir M, and Sepandi M. 2020. Estimate of the Basic Reproduction 569 Number for COVID-19: A Systematic Review and Meta-analysis. J Prev Med Public Health $570 \quad 53: 151-157.10 .3961 / j p m p h .20 .076$

571 41. Ionides EL, Nguyen D, Atchadé Y, Stoev S, and King AA. 2015. Inference for dynamic and 572 latent variable models via iterated, perturbed Bayes maps. Proc Natl Acad Sci U S A 112:719573 724. 10.1073/pnas. 1410597112 
574 42. King AA, Nguyen D, Ionides EL. Statistical inference for partially observed markov

575 processes via the $r$ package pomp. Journal of Statistical Software. 2016;69(1):1-43.

576 43. R Core Team. R: A language and environment for statistical computing [Internet]. Vienna,

577 Austria: R Foundation for Statistical Computing; 2020. Available from: https://www.R-

578 project.org/

579 44. de Vries A, Microsoft. Checkpoint: Install packages from snapshots on the checkpoint server 580 for reproducibility [Internet]. 2020. Available from: https://CRAN.R-

581 project.org/package=checkpoint

582 45. Raue A, Kreutz C, Maiwald T, Bachmann J, Schilling M, Klingmüller U, and Timmer J.

583 2009. Structural and practical identifiability analysis of partially observed dynamical models by

584 exploiting the profile likelihood. Bioinformatics 25:1923--1929.

585 46. Domenech de Cellès M, Magpantay FMG, King AA, and Rohani P. 2018. The impact of past

586 vaccination coverage and immunity on pertussis resurgence. Sci Transl Med 10.

587 10.1126/scitranslmed.aaj1748

588 47. Domenech de Cellès M, Arduin H, Lévy-Bruhl D, Georges S, Souty C, Guillemot D, Watier

589 L, and Opatowski L. 2019. Unraveling the seasonal epidemiology of pneumococcus. Proc Natl

590 Acad Sci U S A 116:1802-1807. 10.1073/pnas. 1812388116

591 48. Maier BF, and Brockmann D. 2020. Effective containment explains subexponential growth

592 in recent confirmed COVID-19 cases in China. Science 368:742-746. 10.1126/science.abb4557

593 49. Pollán M, Pérez-Gómez B, Pastor-Barriuso R, Oteo J, Hernán MA, Pérez-Olmeda M,

594 Sanmartín JL, Fernández-García A, Cruz I, Fernández de Larrea N, Molina M, Rodríguez- 
595 Cabrera F, Martín M, Merino-Amador P, León Paniagua J, Muñoz-Montalvo JF, Blanco F, Yotti 596 R, and ENE-COVID Study Group. 2020. Prevalence of SARS-CoV-2 in Spain (ENE-COVID): a 597 nationwide, population-based seroepidemiological study. Lancet. 10.1016/S0140$598 \quad 6736(20) 31483-5$

599 50. Kucharski AJ, Russell TW, Diamond C, Liu Y, Edmunds J, Funk S, Eggo RM, and Centre 600 for Mathematical Modelling of Infectious Diseases COVID-19 working group. 2020. Early 601 dynamics of transmission and control of COVID-19: a mathematical modelling study. Lancet 602 Infect Dis. 10.1016/S1473-3099(20)30144-4

603 51. Davies NG, Klepac P, Liu Y, Prem K, Jit M, CMMID COVID-19 working group, and Eggo 604 RM. 2020. Age-dependent effects in the transmission and control of COVID-19 epidemics. Nat 605 Med. 10.1038/s41591-020-0962-9

606 52. Bunyavanich S, Do A, and Vicencio A. 2020. Nasal Gene Expression of Angiotensin607 Converting Enzyme 2 in Children and Adults. JAMA. 10.1001/jama.2020.8707

608 53. Jüni P, Rothenbühler M, Bobos P, Thorpe KE, da Costa BR, Fisman DN, Slutsky AS, and 609 Gesink D. 2020. Impact of climate and public health interventions on the COVID-19 pandemic: 610 a prospective cohort study. CMAJ 192:E566-E573. 10.1503/cmaj.200920

611 54. Gaudart J, Landier J, Huiart L, Legendre E, Lehot L, Bendiane MK, Chiche L, Petitjean A, 612 Mosnier E, Kirakoya-Samadoulougou F, Demongeot J, Piarroux R, and Rebaudet S. 2021. 613 Factors associated with the spatial heterogeneity of the first wave of COVID-19 in France: a 614 nationwide geo-epidemiological study. Lancet Public Health. 10.1016/S2468-2667(21)00006-2 
615 55. Sehra ST, Salciccioli JD, Wiebe DJ, Fundin S, and Baker JF. 2020. Maximum Daily

616 Temperature, Precipitation, Ultraviolet Light, and Rates of Transmission of Severe Acute

617 Respiratory Syndrome Coronavirus 2 in the United States. Clin Infect Dis 71:2482-2487.

$618 \quad 10.1093 / \mathrm{cid} / \mathrm{ciaa} 681$

619 56. Baker RE, Yang W, Vecchi GA, Metcalf CJE, and Grenfell BT. 2020. Susceptible supply

620 limits the role of climate in the early SARS-CoV-2 pandemic. Science 369:315-319.

$621 \quad 10.1126 /$ science.abc2535

622 57. Park SW, Cornforth DM, Dushoff J, and Weitz JS. 2020. The time scale of asymptomatic

623 transmission affects estimates of epidemic potential in the COVID-19 outbreak. Epidemics

$62431: 100392.10 .1016 /$ j.epidem.2020.100392

625 58. Dee K, Goldfarb DM, Haney J, Amat JAR, Herder V, Stewart M, Szemiel AM, Baguelin M, 626 and Murcia PR. 2021. Human rhinovirus infection blocks SARS-CoV-2 replication within the 627 respiratory epithelium: implications for COVID-19 epidemiology. J Infect Dis.

628 10.1093/infdis/jiab147

629 59. Ozaras R, Cirpin R, Duman H, Duran A, Arslan O, and Leblebicioglu H. 2020. An open call 630 for influenza vaccination pending the new wave of COVID-19. J Med Virol. 10.1002/jmv.26272

631 60. Lessler J, Reich NG, Brookmeyer R, Perl TM, Nelson KE, and Cummings DAT. 2009.

632 Incubation periods of acute respiratory viral infections: a systematic review. Lancet Infect Dis

633 9:291-300. 10.1016/S1473-3099(09)70069-6 
634 61. Carrat F, Vergu E, Ferguson NM, Lemaitre M, Cauchemez S, Leach S, and Valleron A-J. 635 2008. Time lines of infection and disease in human influenza: a review of volunteer challenge 636 studies. Am J Epidemiol 167:775-785. 10.1093/aje/kwm375

637 62. Kim D, Quinn J, Pinsky B, Shah NH, and Brown I. 2020. Rates of Co-infection Between 638 SARS-CoV-2 and Other Respiratory Pathogens. JAMA. 10.1001/jama.2020.6266

639 63. Marín-Hernández D, Schwartz RE, and Nixon DF. 2020. Epidemiological evidence for 640 association between higher influenza vaccine uptake in the elderly and lower COVID-19 deaths 641 in Italy. J Med Virol. 10.1002/jmv.26120

642 64. Arokiaraj MC. 2020. Correlation of Influenza Vaccination and the COVID-19 Severity. 643 Available at SSRN 3572814.

644 65. Del Riccio M, Lorini C, Bonaccorsi G, Paget J, and Caini S. 2020. The Association between 645 Influenza Vaccination and the Risk of SARS-CoV-2 Infection, Severe Illness, and Death: A 646 Systematic Review of the Literature. Int J Environ Res Public Health 17.

$647 \quad 10.3390 /$ ijerph17217870

648 66. Salem ML, and El-Hennawy D. 2020. The possible beneficial adjuvant effect of influenza 649 vaccine to minimize the severity of COVID-19. Med Hypotheses 140:109752.

$650 \quad 10.1016 /$ j.mehy.2020.109752

651 67. Pinky L, Dobrovolny HM. SARS-CoV-2 coinfections: Could influenza and the common 652 cold be beneficial? J Med Virol. 2020 May; 
653 68. Paget J, Caini S, Cowling B, Esposito S, Falsey AR, Gentile A, Kyncl J, McIntyre C, Pitman

654 R, and Lina B. 2020. The impact of influenza vaccination on the COVID-19 pandemic?

655 Evidence and lessons for public health policies. Vaccine. 10.1016/j.vaccine.2020.08.024

656 69. Domenech de Cellès M, Arduin H, Varon E, Souty C, Boëlle P-Y, Lévy-Bruhl D, van der

657 Werf S, Soulary J-C, Guillemot D, Watier L, and Opatowski L. 2018. Characterizing and

658 Comparing the Seasonality of Influenza-Like Illnesses and Invasive Pneumococcal Diseases

659 Using Seasonal Waveforms. Am J Epidemiol 187:1029-1039. 10.1093/aje/kwx336

660 70. Camacho A, Ballesteros S, Graham AL, Carrat F, Ratmann O, and Cazelles B. 2011.

661 Explaining rapid reinfections in multiple-wave influenza outbreaks: Tristan da Cunha 1971

662 epidemic as a case study. Proc Biol Sci 278:3635-3643. 10.1098/rspb.2011.0300

663 71. Svensson A. 2007. A note on generation times in epidemic models. Math Biosci 208:300-

$664 \quad 311.10 .1016 /$ j.mbs.2006.10.010

665 72. Wood SN. 2010. Statistical inference for noisy nonlinear ecological dynamic systems. Nature 666 466:1102-1104. 10.1038/nature09319

667 


\section{Figure 1}

Potential drivers of SARS-CoV-2 transmission in Belgium, Italy, Norway, and Spain.

A: time plot of the stringency index, a country-level aggregate measure of the number and of the strictness of non-pharmaceutical control measures implemented by governments. The vertical dashed line indicates the start of the nationwide lockdown [17]. B: time plot of influenza incidence, calculated as the product of the incidence of influenza-like illnesses and of the fraction of samples positive to any influenza virus (see also Fig. S1 for a time plot of the latter two variables). The vertical dashed lines delimitate the period of overlap between SARS-CoV-2and influenza, defined as the period between the assumed start date of SARSCoV- 2 community transmission and 6 weeks after the epidemic peak of influenza[68]. In each country, the time series displayed were incorporated as covariates, which modulated the transmission rate of SARS-CoV-2 in our model (see Methods). In B, the $y$-axis values differ for each panel. 
A
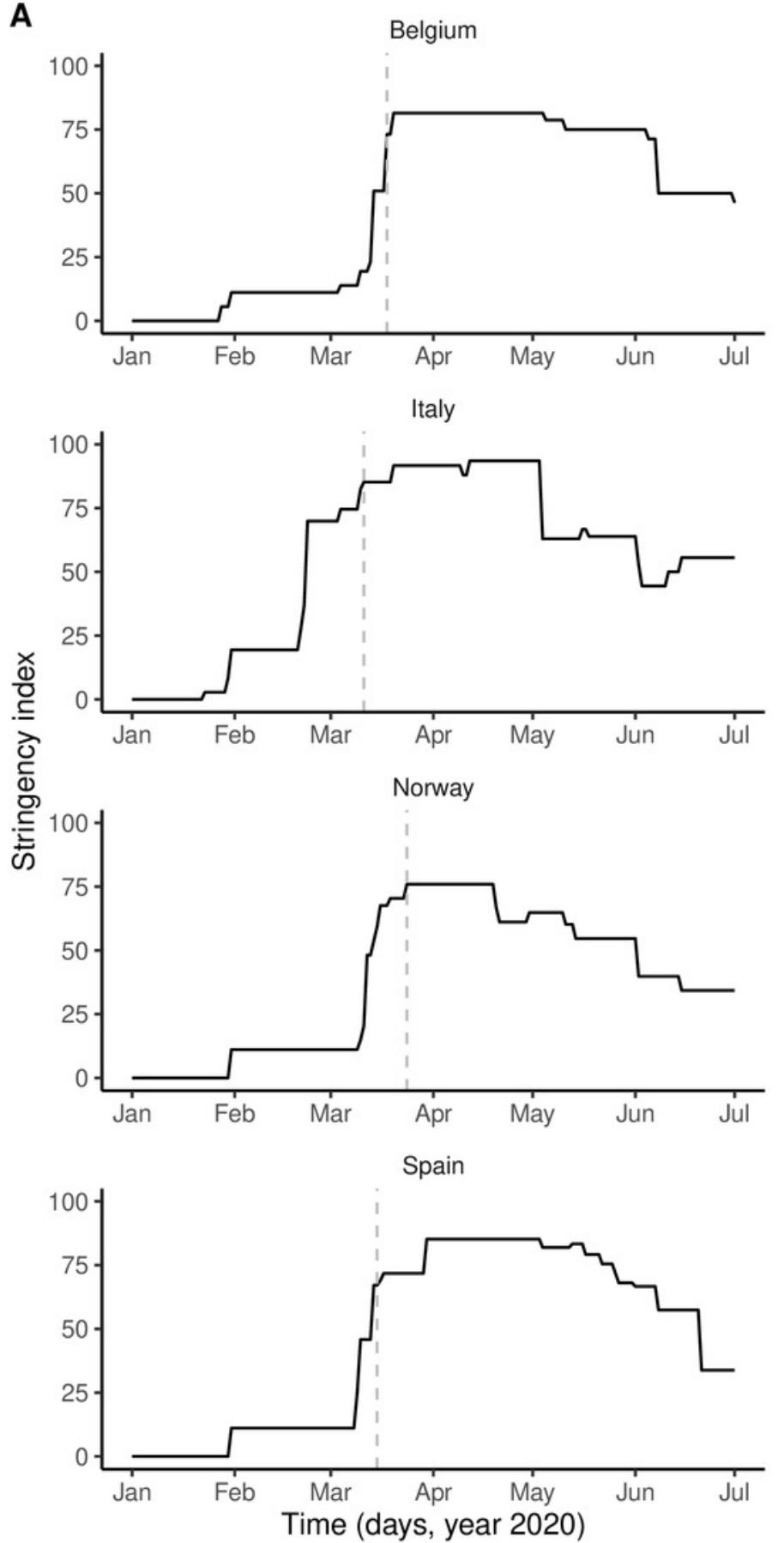

B

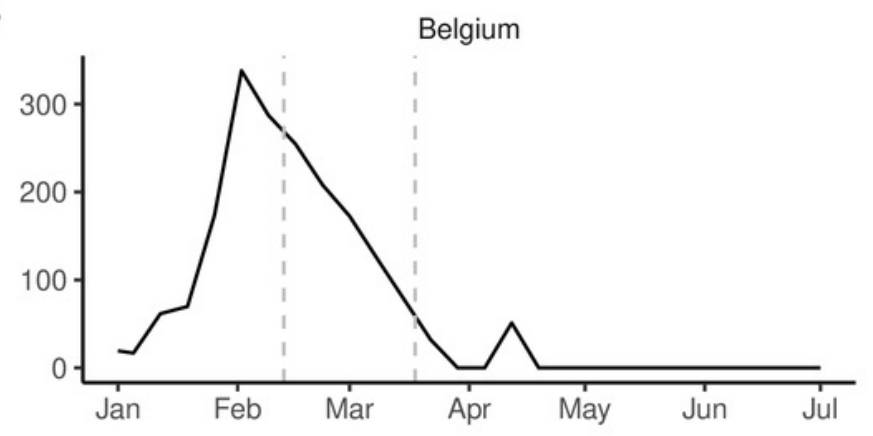

Italy
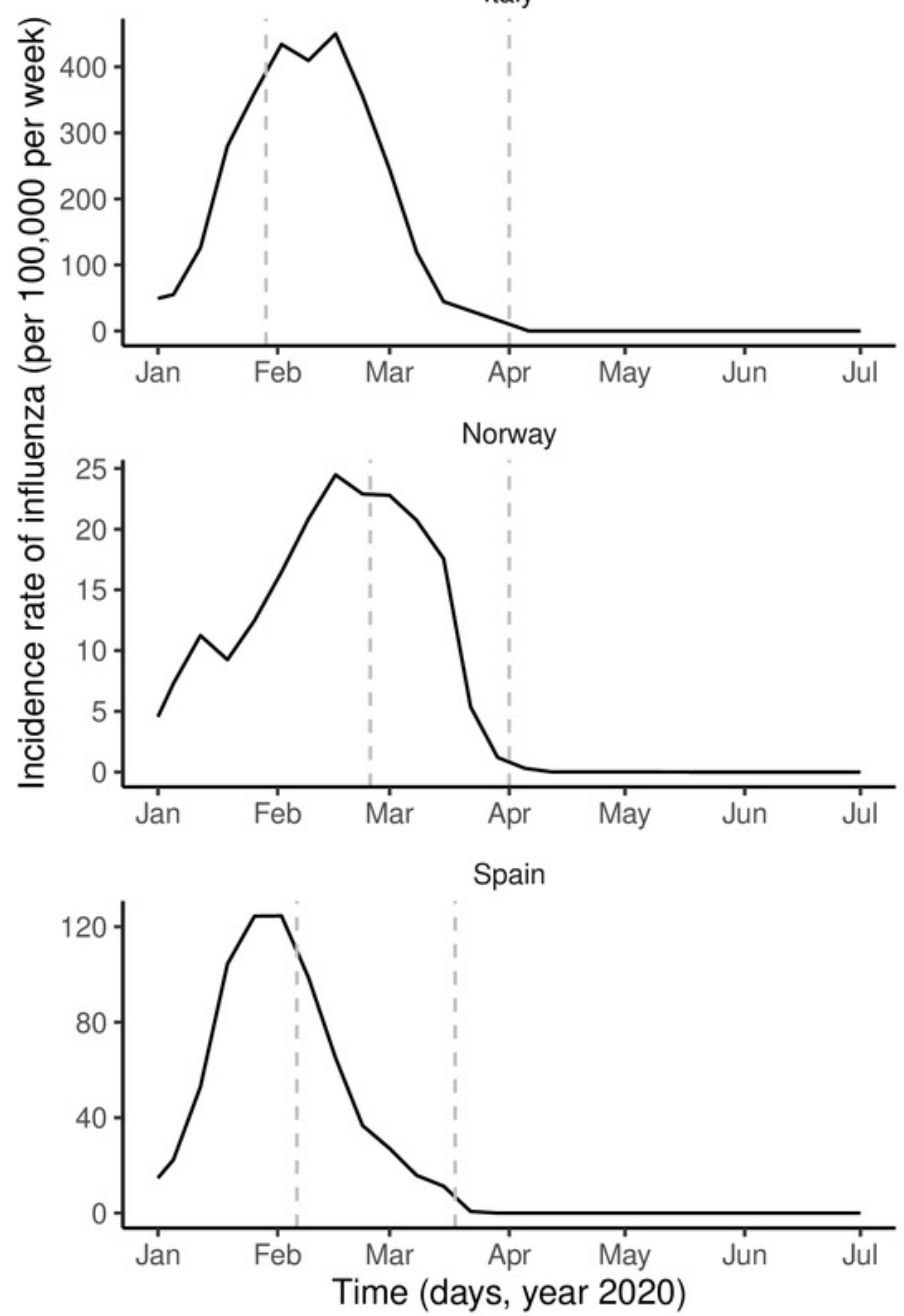


\section{Figure 2}

Dynamics of SARS-CoV-2 transmission and of COVID-19 mortality in Belgium, Italy, Norway, and Spain.

A: time plot of the estimated effective reproductive number $(\mathrm{Re})$. In each panel, the black line represents the maximum likelihood estimate and the grey ribbon the $95 \%$ confidence interval (calculated based on the likelihood profile of the influenza impact parameter, cf. Table2) in each country. The dotted black line represents the effective reproduction number estimated from a model without influenza (i.e., with the influenza impact parameter fixed to 0 and the other parameters estimated from the data). The horizontal grey line is at $\operatorname{Re}=1$. B: time plot of the simulated and observed numbers of daily deaths caused by SARS-CoV-2. In each panel, the light grey lines represent 1,000 model simulations at the maximum likelihood estimate, with one simulation highlighted in dark grey; the black line represents the actual death counts. In A and B, the $x$-axis and the $y$-axis values differ for each panel. 
A
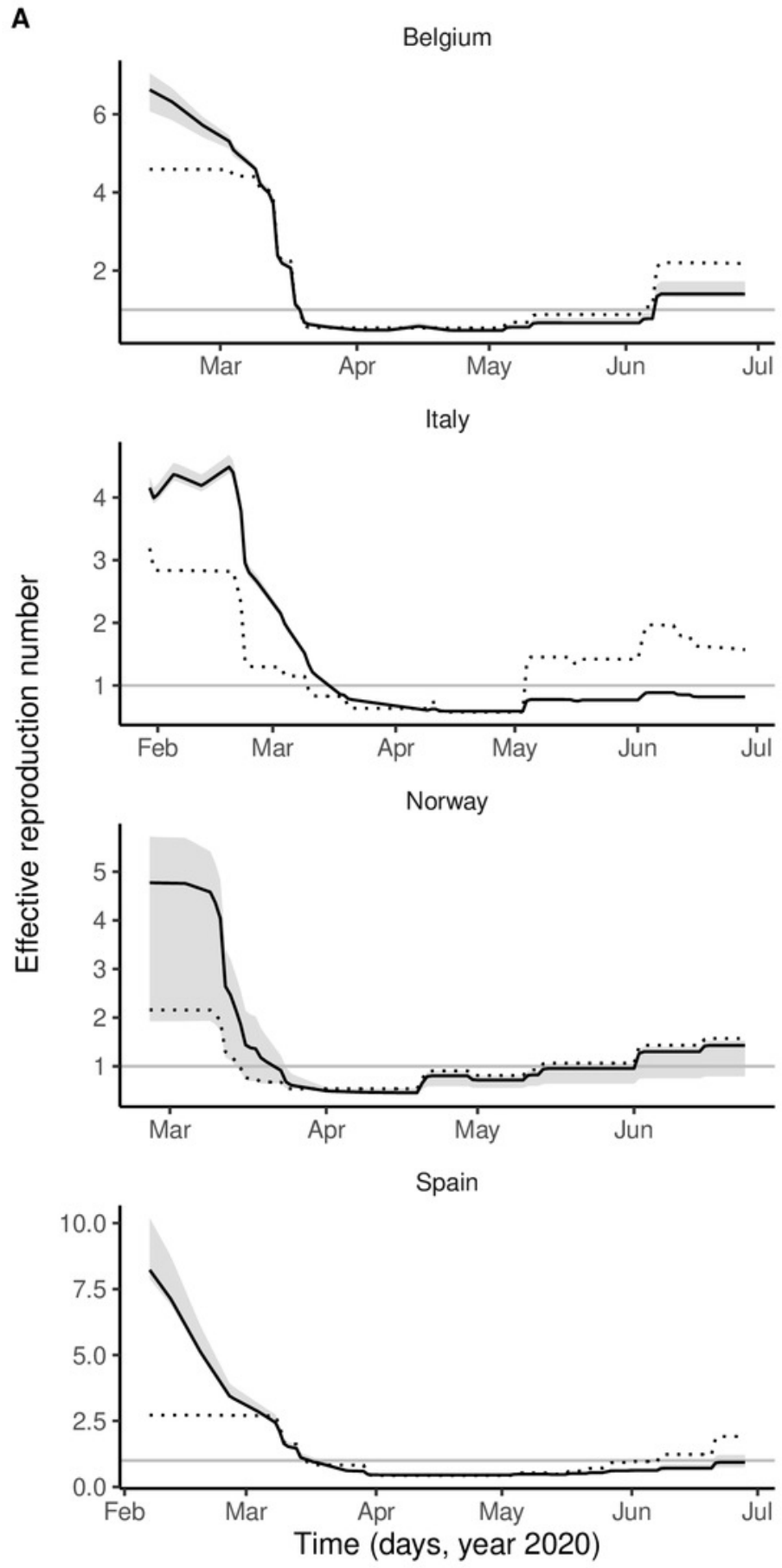

B
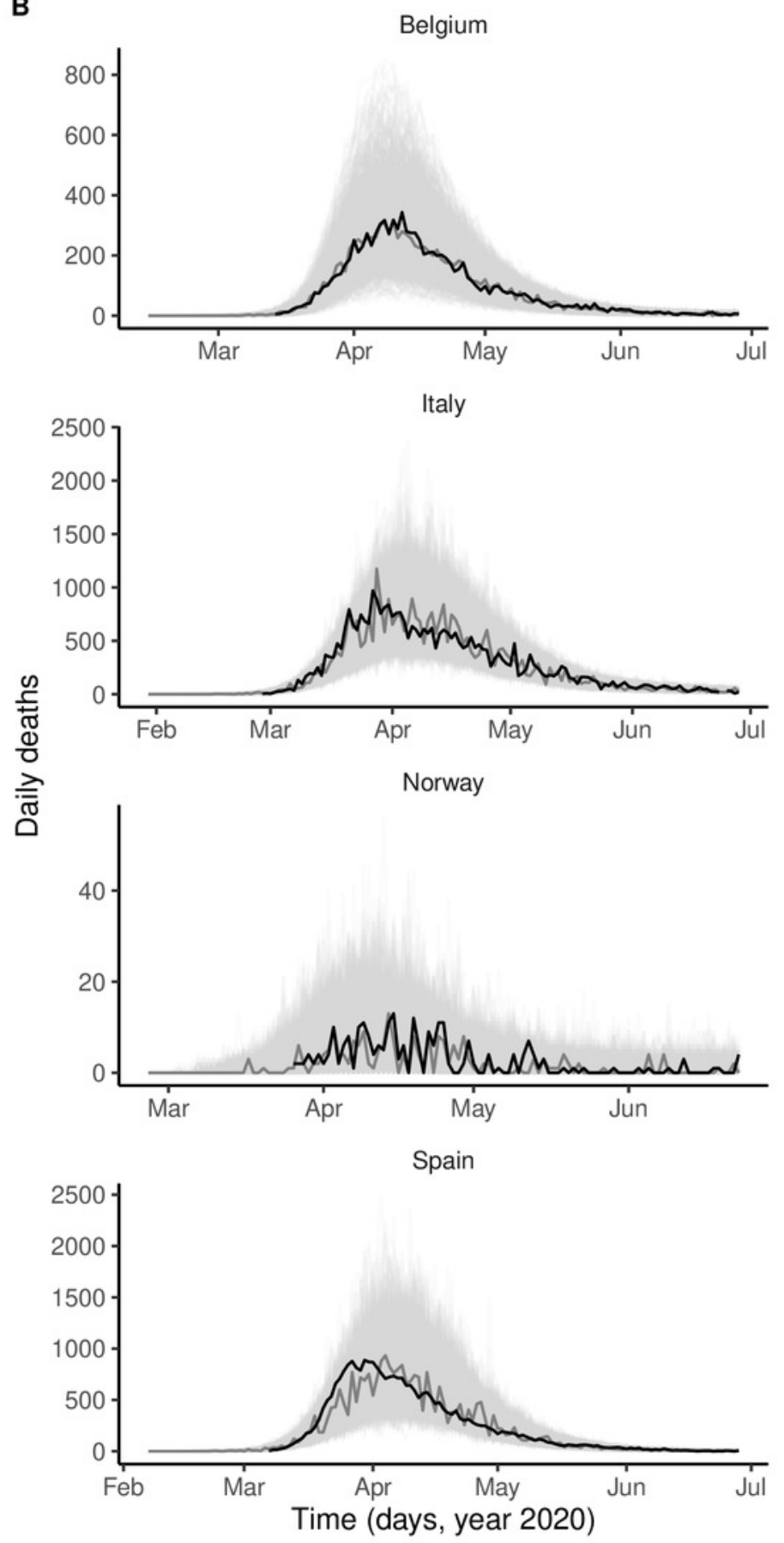
Table $\mathbf{1}$ (on next page)

List of model parameters. 


\begin{tabular}{|c|c|c|c|}
\hline Symbol & Meaning & $\begin{array}{c}\text { Fixed value or } \\
\text { estimation range }\end{array}$ & Comment/Source \\
\hline$D_{E}=1 / \sigma$ & $\begin{array}{c}\text { Average latent } \\
\text { period }\end{array}$ & 4 days & {$[26]$} \\
\hline$D_{I}=1 / \sigma$ & $\begin{array}{l}\text { Average infectious } \\
\text { period }\end{array}$ & 5 days & $\begin{array}{c}\text { Fixed to have } \\
\text { average } \\
\text { generation time of } \\
6.5 \text { days. } \\
\text { Sensitivity analyses: } \\
\text { 2,7days } \\
\end{array}$ \\
\hline$T_{g}=D_{E}+D_{I} / 2$ & $\begin{array}{c}\text { Average generation } \\
\text { time }\end{array}$ & 6.5 days & {$[17,27]$} \\
\hline $1 / \kappa$ & $\begin{array}{l}\text { Average onset-to- } \\
\text { death time }\end{array}$ & 17.8 days & $\begin{array}{c}{[28,17]} \\
\text { Sensitivity analysis: } \\
13 \text { days }\end{array}$ \\
\hline$\mu$ & $\begin{array}{l}\text { Infection- fatality } \\
\text { ratio }\end{array}$ & 0.01 & $\begin{array}{c}{[17,32]} \\
\text { Sensitivity analysis: } \\
0.005\end{array}$ \\
\hline$N$ & Population size & $\begin{array}{l}\text { Belgium: } 11.50 \mathrm{M} \text {; } \\
\text { Italy: } 60.32 \mathrm{M} \text {; } \\
\text { Norway: } 5.37 \mathrm{M} \text {; } \\
\text { Spain: } 47.01 \mathrm{M}\end{array}$ & $\begin{array}{c}2019 \text { demographic } \\
\text { data from the World } \\
\text { Bank }\end{array}$ \\
\hline$s i(t)$ & Stringency index & fixed (covariate) & Fig. 1A \\
\hline$F(t)$ & $\begin{array}{c}\text { Incidence of } \\
\text { influenza (rescaled) }\end{array}$ & fixed (covariate) & Fig. 1B \\
\hline$R_{0}$ & $\begin{array}{c}\text { Basic reproduction } \\
\text { number }\end{array}$ & $1-10$ & {$[40]$} \\
\hline$b$ & $\begin{array}{l}\text { Impact of non- } \\
\text { pharmaceutical } \\
\text { control measures }\end{array}$ & $0.5-2$ & [17] \\
\hline$\beta_{F}$ & $\begin{array}{c}\text { Impact of flu on } \\
\text { SARS-CoV- } \\
2 \text { transmission } \\
\end{array}$ & $\mathbb{R}$ & \\
\hline$k$ & $\begin{array}{c}\text { Dispersion of } \\
\text { individual } \\
\text { reproduction number }\end{array}$ & 0.16 & {$[37,39]$} \\
\hline$k_{D}$ & $\begin{array}{l}\text { Over-dispersion in } \\
\text { death reporting }\end{array}$ & $\mathbb{R}^{+}$ & \\
\hline$E_{1}(0)$ & Initial no exposed & $0-10^{4}$ & Initial condition \\
\hline
\end{tabular}




\section{Table 2 (on next page)}

Model parameter estimates in Belgium, Italy, Norway, and Spain.

For the proportion infected as of May 4, the numbers between parentheses represent a $95 \%$ prediction interval, based on 1,000 simulations at the maximum likelihood estimate. For the other parameters, they represent an approximate 95\% confidence interval, calculated using either the profile likelihood[45] (parameter beta_F ) or a parametric bootstrap (other parameters). SE: standard error, calculated using 5 replicate particle filters, each with 20,000 particles, at the maximum likelihood estimate. 


\begin{tabular}{c|c|c|c|c}
\hline Quantity & Belgium & Italy & Norway & Spain \\
\hline Study period (year 2020) & 13 Feb-28 Jun & 29 Jan-28 Jun & 25 Feb-28 Jun & 06 Feb-28 Jun \\
\hline Log-likelihood (SE) & $-384.4(<0.1)$ & $-649.5(0.1)$ & $-161.8(<0.1)$ & $-558.5(0.2)$ \\
\hline Basic reproduction number $\left(R_{0}\right)$ & $\begin{array}{c}3.4 \\
(2.5,4.1)\end{array}$ & $\begin{array}{c}1.2 \\
(1.1,1.4)\end{array}$ & $\begin{array}{c}2.2 \\
(1.0,2.5)\end{array}$ & $\begin{array}{c}1.4 \\
(1.0,1.9)\end{array}$ \\
\hline $\begin{array}{c}1.03 \\
\text { Impact of control measures }(b)\end{array}$ & $\begin{array}{c}0.53 \\
(0.96,1.07)\end{array}$ & $\begin{array}{c}1.05 \\
(0.50,0.61)\end{array}$ & $\begin{array}{c}0.75 \\
(0.56,0.86)\end{array}$ \\
\hline $\begin{array}{c}\text { Average relative variation in SARS- } \\
\text { CoV-2 transmission rate associated } \\
\text { with influenza }\left(\beta_{F}\right)\end{array}$ & $\begin{array}{c}0.8 \\
(0.5,1.3)\end{array}$ & $\begin{array}{c}1.8 \\
(1.5,2.0)\end{array}$ & $\begin{array}{c}1.0 \\
(0.1,2.0)\end{array}$ & $\begin{array}{c}2.4 \\
(1.7,4.0)\end{array}$ \\
\hline $\begin{array}{c}\text { Initial number exposed to SARS-CoV- } \\
2\left(E_{1}(0)\right)\end{array}$ & $\begin{array}{c}100 \\
(20,200)\end{array}$ & $\begin{array}{c}530 \\
(260,1000)\end{array}$ & $\begin{array}{c}130 \\
(100,2800)\end{array}$ & $\begin{array}{c}400 \\
(170,780)\end{array}$ \\
\hline $\begin{array}{c}7 \times 10^{-4} \\
\text { Over-dispersion in death reporting }\left(k_{D}\right)\end{array}$ & $\begin{array}{c}0.07 \\
1,47) \times\end{array}$ & $\begin{array}{c}0.16 \\
(0.05,0.09)\end{array}$ & $\begin{array}{c}(0.01,0.42) \\
(0.08\end{array}$ \\
\hline $\begin{array}{c}\text { Proportion infected, as of 4 May 2020 } \\
(\%)\end{array}$ & $\begin{array}{c}8.8 \\
(3.7,17.1)\end{array}$ & $\begin{array}{c}5.4 \\
(3.9,7.3)\end{array}$ & $\begin{array}{c}0.4 \\
(0.2,0.8)\end{array}$ & $\begin{array}{c}6.0 \\
(3.8,8.6)\end{array}$ \\
\hline
\end{tabular}

1 